\title{
Nicotine Enhancement of Dopamine Release by a Calcium- Dependent Increase in the Size of the Readily Releasable Pool of Synaptic Vesicles
}

\author{
Timothy J. Turner \\ Department of Neuroscience, Tufts University School of Medicine, Boston, Massachusetts 02111
}

\begin{abstract}
A major factor underlying compulsive tobacco use is nicotine-induced modulation of dopamine release in the mesolimbic reward pathway (Wise and Rompre, 1989). An established biochemical mechanism for nicotine-enhanced dopamine release is by activating presynaptic nicotinic acetylcholine receptors (nAChRs) (Wonnacott, 1997). Prolonged application of $10^{-7}$ to $10^{-5} \mathrm{M}$ nicotine to striatal synaptosomes promoted a sustained efflux of $\left[{ }^{3} \mathrm{H}\right]$ dopamine. This nicotine effect was mediated by non- $\alpha 7 \mathrm{nAChRs,} \mathrm{because} \mathrm{it} \mathrm{was}$ blocked by $5 \mu \mathrm{m}$ mecamylamine but was resistant to $100 \mathrm{~nm} \alpha$-bungarotoxin $(\alpha \mathrm{BgTx})$. Dopamine release was diminished by omitting $\mathrm{Na}^{+}$or by applying peptide calcium channel blockers, indicating that nAChRs trigger release by depolarizing the nerve terminals. However, because $\alpha 7$ receptors rapidly desensitize in the continuous presence of agonists, a repetitive stimulation protocol was used to evaluate the possible significance of desensitization. This protocol produced a transient increase in $\left[{ }^{3} \mathrm{H}\right]$ dopamine released by depolarization and a significant increase in the response to hypertonic solutions that measure the size of the readily releasable pool (RRP) of synaptic vesicles. The nicotine-induced increase in the size of the readily releasable pool was blocked by $\alpha \mathrm{BgTx}$ and by the calmodulin antagonist calmidazolium, suggesting that $\mathrm{Ca}^{2+}$ entry through $\alpha 7 \mathrm{nAChRs} \mathrm{specifically} \mathrm{enhances} \mathrm{synaptic} \mathrm{vesicle} \mathrm{mobilization} \mathrm{at} \mathrm{dopa-}$ mine terminals. Thus, nicotine enhances dopamine release by two complementary actions mediated by discrete nAChR subtypes and suggest that the $\alpha 7 \mathrm{nAChR}$-mediated pathway is tightly and specifically coupled to refilling of the RRP of vesicles in dopamine terminals.
\end{abstract}

Key words: striatum; acetylcholine; presynaptic; kinetics; $\alpha 7 \mathrm{nAChR;} \alpha$-bungarotoxin

\section{Introduction}

Presynaptic ionotropic receptors are gaining recognition for their role in modulating synaptic activity (Miller, 1998; MacDermott et al., 1999). Unlike presynaptic metabotropic receptors that typically diminish the probability of release, presynaptic ionotropic receptor activity generally enhances neurotransmitter release. As observed by recording synaptic events in postsynaptic neurons, the enhancement appears as an increase in the frequency of quantal, or miniature, events. Examples of such enhancement include nicotinic acetylcholine receptor ( $\mathrm{AAChR}$ ) modulation of glutamate release in hippocampus (Gray et al., 1996) and of GABA release in thalamus (Léna and Changeux, 1997), NMDA receptor-mediated glutamate, GABA, and 5-HT release, and 5- $\mathrm{HT}_{3}$ receptor-mediated increases in GABA release in amygdala (Koyama et al., 2000). In addition, biochemical evidence indicates that presynaptic ionotropic receptor activation promotes the release of the monoamines dopamine (Rapier et al., 1988; Grady et al., 1992) and norepinephrine (Clarke and Reuben, 1996).

\footnotetext{
Received April 23, 2004; revised 0ct. 1, 2004; accepted Nov. 5, 2004.

This work was supported by National Science Foundation Grant IBN 0218619. I thank Lorna Colquhoun for her efforts in the early stage of this project. I also thank Kathy Dunlap, Michele Jacobs, and other members of the Department of Neuroscience for their insightful comments.

Correspondence should be addressed to Timothy J. Turner, Department of Neuroscience, Tufts University School of Medicine, 136 Harrison Avenue, Boston, MA 02111. E-mail: timothy.turner@tufts.edu.

DOI:10.1523/JNEUROSCI.1559-04.2004

Copyright $\odot 2004$ Society for Neuroscience ～0270-6474/04/2411328-09\$15.00/0
}

The mechanisms responsible for coupling presynaptic ionotropic receptor activity to an enhanced probability of release remain incompletely understood. On the basis of the biophysical properties of ionotropic receptors, there are at least two likely possibilities to be considered. First, inward current mediated by presynaptic receptors, or secondarily by presynaptic $\mathrm{Na}^{+}$channels, could activate voltage-gated $\mathrm{Ca}^{2+}$ channels to trigger exocytosis. Second, many presynaptic ionotropic receptors have considerable permeability to $\mathrm{Ca}^{2+}$, providing a direct path for $\mathrm{Ca}^{2+}$ entry through the presynaptic receptor. Receptor-mediated $\mathrm{Ca}^{2+}$ entry, through some unidentified intracellular pathway, could lead to increases in the frequency of quantal events.

In this report, I focused on nicotine-stimulated dopamine release from striatal nerve terminals in an effort to differentiate the relative contribution of these two mechanisms to presynaptic modulation of neurotransmitter release. Synaptosomes, a highly reduced preparation enriched in nerve terminals, was used to study presynaptic actions of nicotine in isolation of postsynaptic or "preterminal" effects. The ability of nicotine to modulate $\left[{ }^{3} \mathrm{H}\right]$ dopamine secretion from synaptosomes was measured using two distinct presynaptic activities: nicotine-evoked secretion and hypertonic sucrose-induced exocytosis of the readily releasable pool (RRP) of synaptic vesicles. Consistent with previous reports (Rapier et al., 1988; Grady et al., 1992), prolonged application of nicotine at pharmacologically relevant concentrations produced modest amounts of $\mathrm{Ca}^{2+}$-dependent $\left[{ }^{3} \mathrm{H}\right]$ dopamine 
release that required neuronal calcium channel activity. In addition, two different conditioning protocols were used to demonstrate that nicotine enhanced striatal $\left[{ }^{3} \mathrm{H}\right]$ dopamine release, as assessed by measuring both depolarization-evoked and hypertonic sucrose-induced release. The selective blockade of the nicotine-induced enhancement of the response to sucrose by $\alpha$-bungarotoxin $(\alpha \mathrm{BgTx})$ and by calmidazolium suggests that $\mathrm{Ca}^{2+}$ entry through $\alpha 7 \mathrm{nAChR}$ regulates synaptic vesicle dynamics via a calmodulin-dependent pathway.

\section{Materials and Methods}

For the preparation of synaptosomes, striatal tissue from adult mice (20-30 gm) was dissected and then homogenized in $4 \mathrm{ml}$ of $0.32 \mathrm{M}$ sucrose and 1 mM EDTA in an "AA" Wheaton glass-Teflon homogenizer (Thomas Scientific, Swedesboro, NJ). The supernatant fraction remaining after low-speed centrifugation $(1500 \times \mathrm{g}, 7 \mathrm{~min})$ was divided into two equal portions and applied to two identical discontinuous Percoll (Amersham Biosciences, Piscataway, NJ) gradients, which were centrifuged at $50,000 \times g$ for $5 \mathrm{~min}$ (Dunkley et al., 1986). The material that migrated to the $10 / 15$ and $15 / 23 \%$ Percoll boundaries was collected and washed in basal buffer (in mM: $145 \mathrm{NaCl}, 2.7 \mathrm{KCl}, 1.0 \mathrm{MgCl}_{2}, 10$ glucose, and 10 HEPES-Tris, $\mathrm{pH} 7.4$ ) by two 7 min centrifugation steps at $12,000 \times g$. The final pellet was resuspended in basal buffer and stored on ice until use.

In a typical experiment, a $40 \mu \mathrm{l}$ portion of the synaptosomal suspension containing $\sim 50 \mu \mathrm{g}$ of protein was combined with $10 \mu \mathrm{l}$ of $\left[{ }^{3} \mathrm{H}\right] \mathrm{do}-$ pamine (28.0 Ci/mmol; PerkinElmer Life Sciences, Boston, MA) that had been prepared by evaporating the aqueous stock solution ( $2 \%$ ethanol) under a stream of $\mathrm{N}_{2}$ and dissolving the residue in basal buffer at a concentration of $1 \mu \mathrm{Ci} / \mu$ l. The loading proceeded for $12 \mathrm{~min}$ at a concentration of exogenous dopamine of $\sim 3.6 \mu \mathrm{m}$. The total amount of radioactivity transported was generally $\sim 200,000 \mathrm{cpm} / \mathrm{sample}$, and this transport was blocked by $>95 \%$ by $10 \mathrm{~nm}$ GBR-12935 [(1-[2(diphenylmethoxy)ethyl]-4-(3-phenylpropyl)-piperazine)], a selective inhibitor of the plasma membrane dopamine transporter. The loading reaction was stopped by adding $750 \mu \mathrm{l}$ of basal buffer that contained 1 $\mathrm{mg} / \mathrm{ml} \mathrm{BSA}$, which improved retention of the synaptosomes on the filter. This suspension was applied to a filtration sandwich composed of cellulose ester and glass fiber filters, as described previously (Turner et al., 1989).

Release was measured using a superfusion device in conjunction with a fraction collector modified from a phonograph turntable (Forbush, 1984; Turner and Goldin, 1989). Synaptosomes were superfused with an appropriate stimulus buffer, and fractions ranging from $15 \mathrm{msec}$ (78 $\mathrm{rpm})$ to $100 \mathrm{msec}(12 \mathrm{rpm}$ ) were collected on the turntable. Alternatively, longer fractions were collected by advancing the turntable platter manually, using a quartz metronome (Seiko Corporation, Mahwah, NJ) for timing. Radioactivity in each fraction and the amount remaining on the filter at the end of the experiment were determined by adding $1.5 \mathrm{ml}$ of liquid scintillation mixture (BioSafe II; Research Products, Mt. Prospect, IL) and counting in a Tri-Carb 2100TR liquid scintillation analyzer (Packard Bioscience, Pangbourne, UK).

Stimulus buffers designed to depolarize the synaptosomal membrane were prepared by combining two solutions, the basal buffer and an otherwise identical $\mathrm{KCl}$ solution in which $\mathrm{NaCl}$ had been substituted with $\mathrm{KCl}$ (final $\mathrm{KCl}$ concentration was $147.7 \mathrm{~mm}$ ). These solutions were combined in the appropriate ratio to yield a stimulus buffer with the desired $\mathrm{KCl}$ concentration. $\mathrm{Ca}^{2+}$-dependent release was calculated as the net difference between release at a given $\mathrm{Ca}^{2+}$ concentration and " $\mathrm{Ca}^{2+}$ independent" release evoked with a stimulus buffer that contained no added $\mathrm{Ca}^{2+}$ (free $\left[\mathrm{Ca}^{2+}\right] \sim 3 \mu \mathrm{M}$ ). The small $\mathrm{Ca}^{2+}$-independent component of release has been shown previously to be mediated by thermodynamic reversal of the $\mathrm{Na}^{+}$-dependent transporter that normally concentrates dopamine in the nerve terminal cytoplasm under resting ionic conditions. Data were analyzed using Microsoft (Seattle, WA) Excel. Results were expressed as the ratio of counts per minute in each fraction to the total radioactivity remaining on the filter $(\times 100 \%)$; error bars represent the SEM. Data reported are the average of at least three separate experiments performed on different days with freshly prepared synapto-
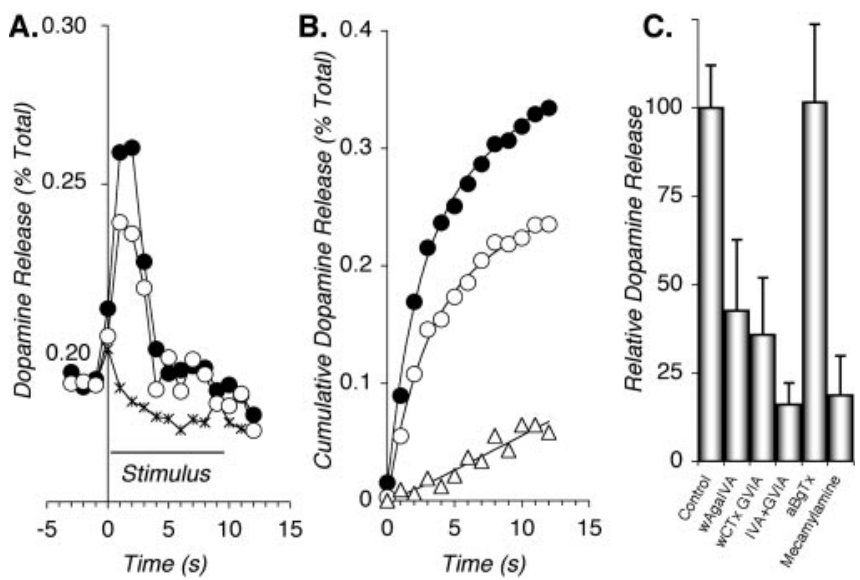

Figure 1. Prolonged nicotine application evokes depolarization-dependent dopamine release. Striatal synaptosomes were loaded with $\left[{ }^{3} \mathrm{H}\right]$ dopamine and placed in a superfusion device to measure the kinetics of dopamine efflux. $A$, The synaptosomes were superfused for $6 \mathrm{sec}$ with basal saline that contained $2.5 \mathrm{~mm} \mathrm{Ca}^{2+}$ to establish a baseline for the efflux rate. At the time indicated as $t=0$, the superfusate was switched to stimulus buffer composed of an identical solution $\left({ }^{*}{ }^{*}\right)$ or one that contained $1 \mu \mathrm{m}$ nicotine $(\mathbf{O} ; n=16)$ or $10 \mu \mathrm{m}$ acetylcholine $(\bigcirc ; n=9)$. Agonist was applied continuously for $10 \mathrm{sec}$, followed by 2 sec of wash with the $\mathrm{Ca}^{2+}$-containing basal saline. $B$, Cumulative, agonist-stimulated release was calculated for nicotine ( $)$ and acetylcholine $(\bigcirc)$ by subtracting the baseline release rate determined in the absence of agonist from the total release (as shown in $A$ ) and by integrating the net release over time. The data were fit to the sum of an exponential plus a linear component (smooth curves). The rate constants for the exponential component derived from the fit $\left(0.44\right.$ and $0.36 \mathrm{sec}^{-1}$ for nicotine and $A C h$, respectively) were not statistically different. When $\mathrm{Na}^{+}$was omitted from the nicotine-containing stimulus buffer, the exponential component was eliminated, leaving only the linear phase $(\triangle) . C$, Pharmacological analysis was performed by preincubating the synaptosomes in $1 \mu \mathrm{m} \omega$ Aga IVA, $1 \mu \mathrm{m} \omega$ CTx GVIA, $1 \mu \mathrm{m} \omega$ Aga IVA plus $1 \mu \mathrm{m} \omega \mathrm{CTx}$ GVIA, 100 $\mathrm{nm} \alpha \mathrm{BgTx}$, or $5 \mu \mathrm{m}$ mecamylamine. The toxin-treated samples were stimulated using a $10 \mathrm{sec}$ application of $1 \mu \mathrm{m}$ nicotine, and cumulative, nicotine-stimulated release was determined as described above. For mecamylamine, $5 \mu \mathrm{m}$ antagonist was also included in the nicotinecontaining stimulation buffer, because this antagonist has relatively rapid kinetics of dissociation. Data shown are the average \pm SEM values obtained in five separate experiments.

somes. To account for any time-dependent changes in release rates, the order of the experimental conditions was randomized for each experiment.

The peptide toxins were prepared as $1.0 \mathrm{~mm}$ stock solutions in water, divided into $10 \mu \mathrm{l}$ portions, and stored at $-70^{\circ} \mathrm{C}$. Working stocks were prepared by diluting the concentrated solutions 10 -fold with basal buffer that contained $1 \mathrm{mg} / \mathrm{ml}$ bovine serum albumin. These working solutions could be subjected to multiple freeze-thaw cycles without apparent loss of activity. $\alpha$-Bungarotoxin was obtained from Sigma (St. Louis, MO), $\omega$-agatoxin IVA ( $\omega$ Aga IVA) was purchased from Peptides International (Frankfort, KY), and $\omega$-conotoxin GVIA ( $\omega \mathrm{CTx}$ GVIA) was purchased from Bachem (Torrance, CA). Other reagents, unless indicated otherwise, were purchased from Fluka (Neu-Ulm, Germany).

\section{Results}

\section{Nicotine depolarizes synaptic terminals}

A biochemical approach was used to study nicotine-evoked dopamine secretion in mouse brain, using striatal synaptosomes metabolically labeled with $\left[{ }^{3} \mathrm{H}\right]$ dopamine. $\left[{ }^{3} \mathrm{H}\right]$ Dopamine release was measured using a superfusion device that combined high flow $(2 \mathrm{ml} / \mathrm{sec})$ through a small chamber $(50 \mu \mathrm{l})$ to provide subsecond temporal resolution $(\tau \approx$ volume/flow $=25 \mathrm{msec})$ (Turner et al., 1993) (Fig. 1). The synaptosomes were superfused with a basal buffer that contained $2.5 \mathrm{~mm} \mathrm{Ca}^{2+}$, and basal efflux rates of $\left[{ }^{3} \mathrm{H}\right]$ dopamine were measured at $1 \mathrm{sec}$ intervals. After 6 $\sec$ (where $t$ is indicated as 0 ), the superfusate was switched to a stimulation buffer that contained $1 \mu \mathrm{M}$ nicotine or $10 \mu \mathrm{M}$ acetylcholine. Both agonists produced a rapid, long-lasting increase in 
the rate of dopamine release. The net, nicotine-stimulated dopamine release rate, calculated by subtracting the basal rate obtained in the absence of agonist, reached a peak within $1 \mathrm{sec}$ of agonist application and subsequently decreased to a slowly decaying plateau. The cumulative release calculated by summing the release over time (Fig. $1 \mathrm{~B}$ ) was well described as the sum of an exponential and linear process. When the nicotine-containing stimulus buffer had $\mathrm{Na}^{+}$replaced by the impermeant cation $\mathrm{N}$-methylglucammonium ${ }^{+}$, the exponential phase of the fit was eliminated, leaving only the slow linear component.

Pharmacological experiments were performed that were designed to address the mechanisms that underlie nicotine-evoked release from striatal terminals (Fig. $1 C$ ). Mecamylamine at $10 \mu \mathrm{M}$, a nicotinic antagonist that selectively blocks $\alpha \mathrm{BgTx}$-insensitive nAChRs at this concentration (McQuiston and Madison, 1999), inhibited nicotine-evoked dopamine release by $81 \pm 11 \%(n=$ 12). Conversely, $100 \mathrm{~nm} \alpha \mathrm{BgTx}$, the $\alpha 7$-selective toxin, had no effect. These results argue that nicotine-stimulated dopamine release was mediated by non- $\alpha 7 \mathrm{nAChR}$ subtype(s). The $\mathrm{Na}^{+}$requirement (Fig. $1 B$ ) suggested that membrane depolarization was a requisite element of nicotine action. To confirm that voltage-gated $\mathrm{Ca}^{2+}$ channels were involved (Prince et al., 1996), synaptosomes were pretreated with selective antagonists of neuronal calcium channels to determine whether they could inhibit nicotine-stimulated dopamine release. The P/Q channel antagonist $\omega$ Aga IVA $(1 \mu \mathrm{M})$ and the $\mathrm{N}$ channel blocker $\omega$ CTx GVIA (1 $\mu \mathrm{M})$ each reduced nicotine-stimulated release, by 57 and $64 \%$, respectively. When both peptides were combined, nicotine action was inhibited by $84 \%$. Together, these experiments indicate that prolonged nicotine exposure stimulates dopamine secretion by activating non- $\alpha 7$ nAChRs, causing depolarization-gated calcium entry through neuronal calcium channels that trigger exocytosis.

\section{Repetitive stimulation with nicotine reveals a role for the $\alpha 7$ nAChR subtype}

Despite the evidence that non- $\alpha 7$ receptors were the sole mediators of nicotine in triggering striatal dopamine release, it remained possible that $\alpha 7 \mathrm{nAChR}$ activity might be obscured by rapid receptor desensitization caused by the prolonged application of agonist. To evaluate this hypothesis, a repetitive stimulation protocol was used based on the work of Radcliffe and Dani (1998). The objective of this approach was to activate $\alpha 7$ receptors with a brief pulse of nicotine, allowing sufficient time for the receptors to recover from desensitization before the next pulse was applied. The protocol consisted of a series of four $500 \mathrm{msec}$ depolarizing pulses (using $60 \mathrm{~mm} \mathrm{KCl}$ ) that allowed us to measure the amount of dopamine secretion triggered by neuronal $\mathrm{Ca}^{2+}$ channels. The first two depolarizing pulses were delivered at $20 \mathrm{sec}$ intervals, an interpulse interval that was sufficient to maintain a constant response to $\mathrm{KCl}$ depolarization (Turner and Goldin, 1989). One second after the second depolarizing pulse, a series of five $200 \mathrm{msec}$ conditioning pulses containing $3 \mu \mathrm{M}$ nicotine (or basal saline as a control) was delivered at $10 \mathrm{sec}$ intervals. One second after the fifth conditioning pulse, another pair of $60 \mathrm{~mm} \mathrm{KCl}$-containing depolarizing pulses was delivered at $20 \mathrm{sec}$ intervals to assess any change in the amount of depolarization-evoked dopamine release that was produced by the conditioning protocol. As a control, basal buffer was used as the stimulus solution in the conditioning protocol. Under this condition, there was no change in dopamine release produced by the third or fourth depolarizing pulse (Fig. 2). However, when basal buffer plus $3 \mu \mathrm{M}$ nicotine was used, dopamine release

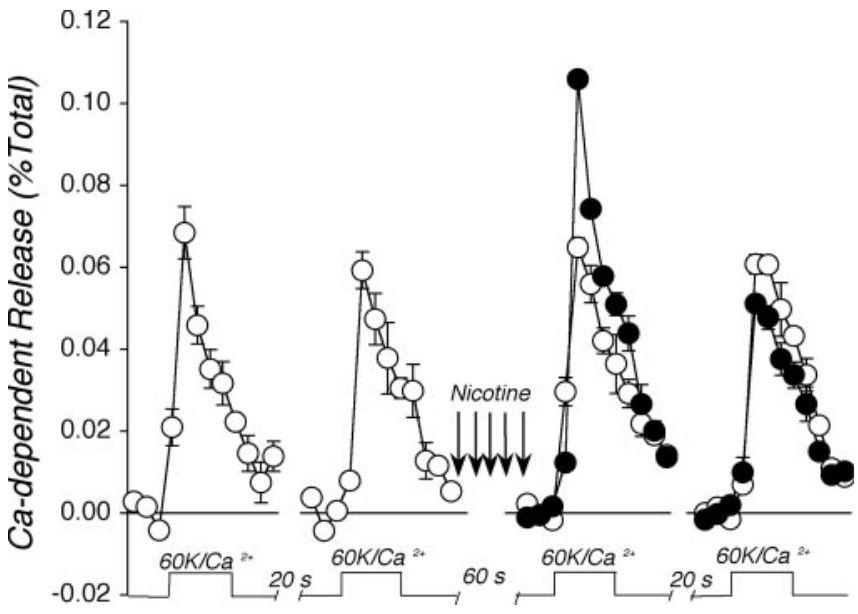

Figure 2. Nicotine enhances evoked release of dopamine. Synaptosomes were depolarized at $20 \mathrm{sec}$ intervals using 500 msec pulses with $60 \mathrm{~mm} \mathrm{~K}^{+}$and $1 \mathrm{~mm} \mathrm{Ca}^{2+}$ to evoke $\mathrm{Ca}^{2+}$ depedent release of $\left[{ }^{3} \mathrm{H}\right]$ dopamine. The net, $\mathrm{Ca}^{2+}$-dependent release was calculated by subtracting release measured in the absence of $\mathrm{Ca}^{2+}$. After the second depolarizing pulse, a series of five $200 \mathrm{msec}$ conditioning pulses, containing $3 \mu \mathrm{m}$ nicotine or saline as control, were delivered at 10 sec intervals. One second after the fifth conditioning pulse, a third depolarizing pulse was delivered, followed 20 sec later by a fourth depolarizing pulse. Synaptosomes that had been exposed to the brief nicotine pulses (O) showed a $44.5 \pm 5.2 \%(n=8)$ increase in the $\mathrm{Ca}^{2+}$-dependent dopamine release, whereas there was no effect of the control conditioning pulses without nicotine (O). The enhancement produced by nicotine was transient, because the response to the fourth depolarizing pulse was slightly depressed ( $84.4 \pm 7.2 \%$ ) relative to the control. Results are expressed as the mean \pm SEM of four experiments performed in triplicate.

evoked by the third depolarizing pulse was significantly increased $(44.5 \%$ increase; $p<0.02 ; n=8)$. This enhancement was transient, because dopamine release during the fourth depolarizing pulse was slightly reduced ( $84.4 \%$ of control values). This result suggested that brief pulses with nicotine transiently enhanced $\left[{ }^{3} \mathrm{H}\right]$ dopamine release.

Nicotine enhances dopamine release by altering the size of the readily releasable pool of synaptic vesicles

In addition to triggering exocytosis, $\mathrm{Ca}^{2+}$ influences several other steps in the synaptic vesicle cycle. Specifically, moderate increases in $\mathrm{Ca}^{2+}$ concentrations have been shown to enhance the size of the RRP of vesicles that are docked and fusion competent in chromaffin cells (Smith et al., 1998) and accelerates the refilling of the RRP in autaptic neurons (Stevens and Wesseling, 1998). Thus, $\mathrm{Ca}^{2+}$ entry via nAChRs could modulate the equilibrium between the reserve and readily releasable pools of synaptic vesicles, enhance refilling by activating endocytosis, or regulate some other unidentified process. To test whether the brief pulses with nicotine enhanced dopamine release by modulating synaptic vesicle dynamics, the size of the RRP was measured by applying saline made hypertonic by the addition of $0.5 \mathrm{M}$ sucrose. This technique has been used previously to measure the size of the RRP of glutamatergic vesicles in synaptosomes (Lonart and Südhof, 2000) and in autaptic cultures (Stevens and Tsujimoto, 1995; Rosenmund and Stevens, 1996).

The validity of using hypertonic sucrose to measure the RRP of dopaminergic vesicles in synaptosomes was evaluated by comparing the properties of sucrose-evoked $\left[{ }^{3} \mathrm{H}\right]$ dopamine release with the results observed previously (Fig. 3). The kinetics of sucrose-evoked release of $\left[{ }^{3} \mathrm{H}\right]$ dopamine were remarkably similar to those observed in autaptic cultures of hippocampal neurons. Superfusion of synaptosomes with saline containing $0.5 \mathrm{M}$ 

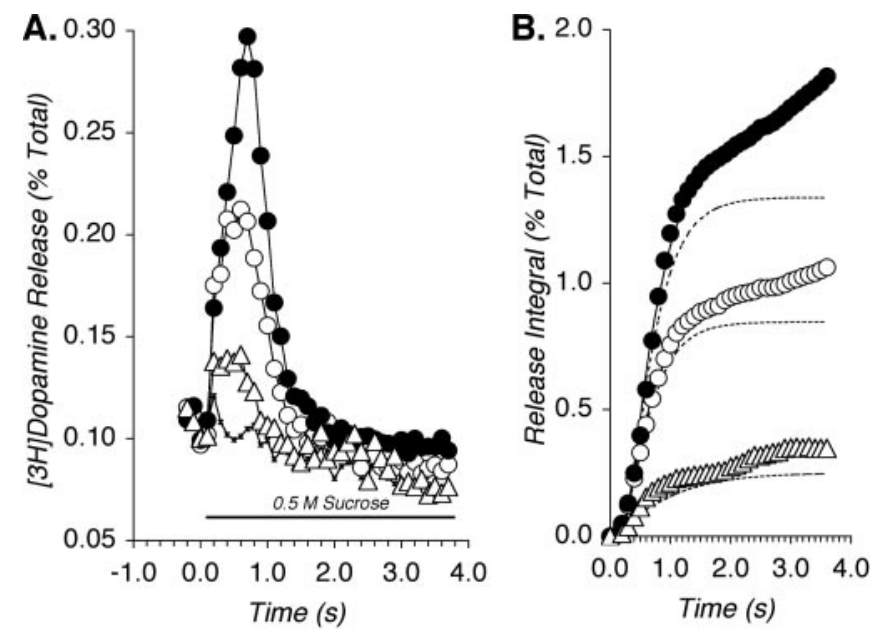

Figure 3. Hypertonic sucrose stimulates secretion of synaptosomal dopamine. $A$, Striatal synaptosomes were loaded with $\left[{ }^{3} \mathrm{H}\right]$ dopamine and superfused with basal saline for $1.8 \mathrm{sec}$ to establish baseline release rates. At the time indicated by the horizontal bar, the superfusate was switched to saline made hypertonic by the addition of $0.5 \mathrm{~m}$ sucrose. The release rate from control synaptosomes $(O)$ increased gradually to a maximum rate observed after $\sim 500 \mathrm{msec}$, and it subsequently decayed within 2 sec to a sustained plateau rate when compared with synaptosomes continuously superfused with isotonic basal saline $\left({ }^{*}{ }^{*}\right)$. Pretreating synaptosomes with $3 \mu \mathrm{MPBu}(-)$ resulted in an increase in the amount of dopamine release, whereas pretreatment with $10 \mu \mathrm{m}$ reserpine $(\triangle)$ greatly diminished sucrose-evoked release. $B$, The net sucrose-evoked release was integrated and fit to the sum of an exponential component plus a linear phase (smooth lines); the amplitude of the exponential component (dashed lines), defined as the RRP, was increased by $57 \%$ by PDBu treatment and decreased by $71 \%$ by reserpine treatment. Data are averages of three experiments performed in quadruplicate.

sucrose produced a gradual increase in the rate of $\left[{ }^{3} \mathrm{H}\right]$ dopamine release that reached a peak within $500 \mathrm{msec}$ and decayed over $\sim 2$ sec to a sustained plateau level (Fig. $3 A$ ). The cumulative release was adequately described as the sum of an exponential component and a linear component. The amplitude of exponential component was defined as the readily releasable pool size. This parameter was appropriately modulated by 12,13-phorbol dibutyrate $(\mathrm{PDBu})$, an activator of protein kinase $\mathrm{C}$ that has been shown to increase the size of the RRP of glutamatergic vesicles in hippocampal neurons (Stevens and Sullivan, 1998) and secretory granules in chromaffin cells (Gillis et al., 1996). After phorbol treatment, the RRP of $\left[{ }^{3} \mathrm{H}\right]$ dopamine was increased by $57 \%$, quantitatively similar to the increase in glutamate release observed at glutamatergic autapses and in chromaffin cells. Finally, treatment with $10 \mu \mathrm{M}$ reserpine, an inhibitor of the $\mathrm{H}^{+}$dependent vesicular monoamine transporter required for dopamine storage in synaptic vesicles (Schuldiner, 1994), markedly diminished the size of the RRP, indicating that sucrose-induced release of transmitter is vesicular in origin.

To determine whether $\mathrm{Ca}^{2+}$ influx through nAChRs might regulate the size of the RRP, nicotine was delivered using repetitive stimulation (five $200 \mathrm{msec}$ pulses at $10 \mathrm{sec}$ intervals), followed $1 \mathrm{sec}$ after the end of the final conditioning pulse by a test pulse with $0.5 \mathrm{M}$ sucrose to measure the size of the RRP (Fig. $4 \mathrm{~A}$ ). The conditioning protocol significantly increased (41.9 $\pm 6.3 \%$; $p<0.02$ ) dopamine release evoked by a hypertonic sucrose test pulse. The increase was quantitatively similar to the effect of the conditioning protocol on $\mathrm{KCl}$-evoked release (Fig. 2). The pharmacology of nicotinic receptors involved in the enhanced response to sucrose was investigated by using the nAChR antagonists mecamylamine and $\alpha \mathrm{BgTx}$. One set of synaptosomes was preincubated with $100 \mathrm{~nm} \alpha \mathrm{BgTx}$ before the nicotine condition-
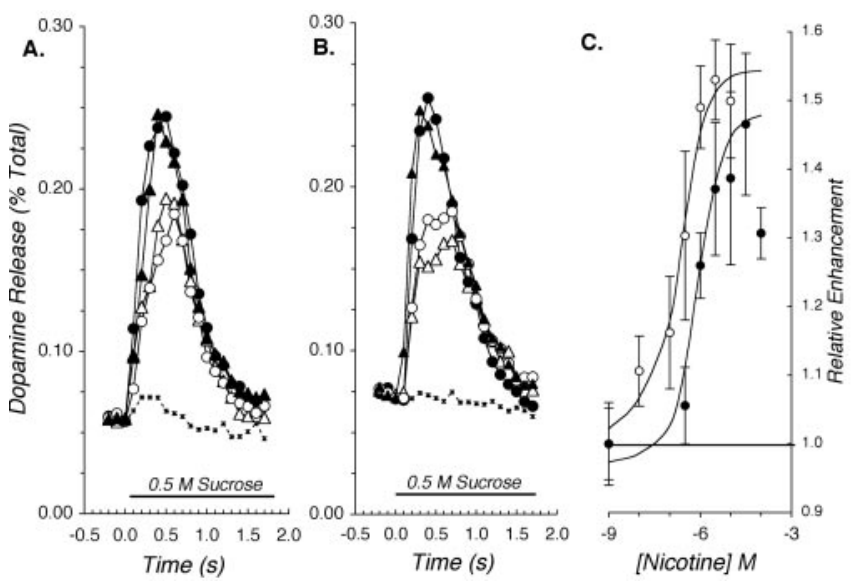

Figure 4. Nicotine enhances the size of the RRP in dopaminergic terminals. The ability of nAChRs to modulate synaptic vesicle dynamics in dopaminergic terminals was assessed by applying five 200 msec conditioning pulses at 10 sec intervals, followed 1 sec after the end of the fifth nicotine pulse by a test pulse that contained $0.5 \mathrm{~m}$ sucrose to measure the size of the RRP.A, At $t=0$, the superfusate was switched to an identical solution $\left({ }^{*}-^{*}\right)$ to measure baseline release rates or a hypertonic saline containing $0.5 \mathrm{~m}$ sucrose. When $3 \mu \mathrm{m}$ nicotine was included in the conditioning pulses ( $)$, the size of the RRP (assessed as described in Fig. 2) was increased by $41.9 \pm 6.3 \%$ when compared with control samples in which no nicotine was included in the conditioning pulses $(\bigcirc)$. The effect of nicotine was essentially blocked when the synaptosomes were pretreated with $100 \mathrm{~nm} \alpha \mathrm{Bg}$ Tx before the nicotine exposure $(\triangle)$. In contrast, the non- $\alpha 7$ antagonist mecamylamine ( $5 \mu \mathrm{m}$ in both the preincubation and conditioning solutions) had no effect against nicotine-induced increases in the $\operatorname{RRP}(\boldsymbol{\Delta}) . B$, The same conditioning protocol was used to evaluate the $\mathrm{Ca}^{2+}$ requirement for nicotine-induced increases in the size of the RRP. Compared with control (O), $3 \mu \mathrm{m}$ nicotine increased the size of the RRP by $40 \%$ when $1 \mathrm{~mm}$ $\mathrm{Ca}^{2+}$ was included in the conditioning solution (O). Incubating the synaptosomes in saturating concentrations (1 $\mu \mathrm{m}$ each) of neuronal calcium channels blockers $\omega$-conotoxin GVIA and $\omega$-conotoxin MVIIC $(\boldsymbol{\Delta})$ had no significant effect on the ability of nicotine to potentiate the sucrose response, but $\mathrm{Ba}^{2+}$ substitution for $\mathrm{Ca}^{2+}$ in the nicotine conditioning pulses abolished the effect $(\triangle)$. Data were averaged from three experiments performed in quadruplicate. $C$, Concentration-response data were obtained for nicotine-evoked release $(\bigcirc$, calculated as described in Fig. 1) or for the enhanced response to sucrose induced by the conditioning protocol (1) as described above) and plotted on the same axis for comparison. Results are the mean \pm SEM of 10 and 6 independent measurements, respectively.

ing protocol, whereas a second set was conditioned with $200 \mathrm{msec}$ pulses that contained both $3 \mu \mathrm{M}$ nicotine and $5 \mu \mathrm{M}$ mecamylamine, a concentration of antagonist that is relatively selective for non- $\alpha 7$ nAChRs (McQuiston and Madison, 1999). The enhanced response to sucrose produced by nicotine was essentially blocked in $\alpha \mathrm{BgTx}$-treated synaptosomes, whereas mecamylamine, at a concentration that blocked nicotine-evoked release of dopamine, had no significant effect.

The effect of nicotine on the response to sucrose was strictly $\mathrm{Ca}^{2+}$ dependent (Fig. 4B). When the $\mathrm{Ca}^{2+}$ in the conditioning stimulus was replaced by $\mathrm{Ba}^{2+}$, there was no nicotine-induced enhancement despite the fact that $\alpha 7 \mathrm{nAChRs}$ are quite permeant to $\mathrm{Ba}^{2+}$ (Sands et al., 1993). Unlike the nicotine-evoked release of $\left[{ }^{3} \mathrm{H}\right]$ dopamine, neuronal calcium channels were not involved in the nicotine-induced enhancement of the sucrose-evoked release. This action of nicotine was unaltered by the combination of $\omega$ CTx GVIA and $\omega$ CTx MVIIC ( $1 \mu \mathrm{M}$ each), inhibitors of $\mathrm{N}$ and $\mathrm{P} / \mathrm{Q}$ calcium channels that strongly reduced nicotine-evoked release.

The relative potency of nicotine in triggering dopamine release (persistent agonist application) and in enhancing the size of the RRP (pulsatile application) was compared to further distinguish the properties of these two activities (Fig. 4C). Concentration-response data indicated an $\mathrm{EC}_{50}$ value for nicotine of 

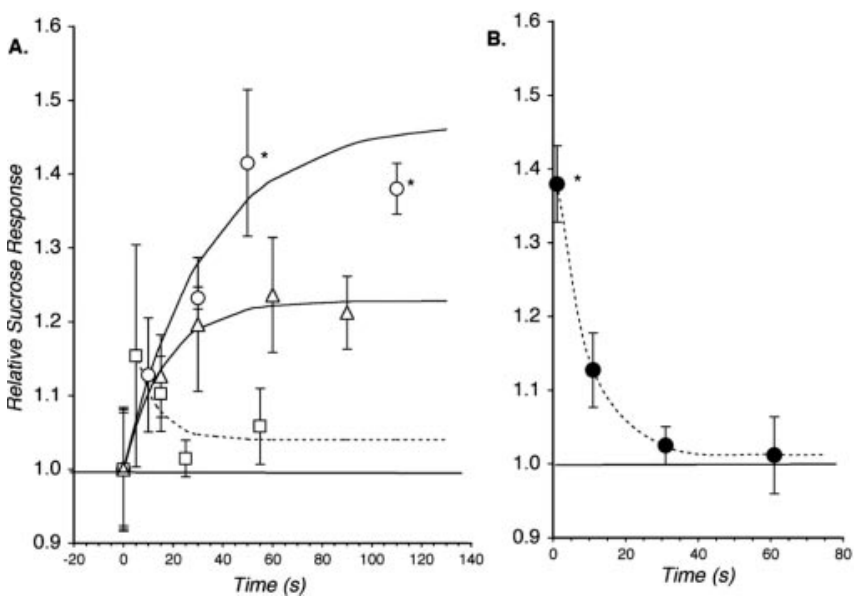

Figure 5. Short-term enhancement of the RRP by nicotine. $A$, The kinetics of the onset of nicotine-induced enhancement were investigated by delivering a defined number of conditioning pulses ( $200 \mathrm{msec}$, with or without $3 \mu \mathrm{m}$ nicotine, $1 \mathrm{~mm} \mathrm{Ca}^{2+}$ ) at $5(\square), 10(\bigcirc)$, or $15(\triangle)$ sec intervals. For each data set, the $0.5 \mathrm{~m}$ sucrose test pulse followed $1.0 \mathrm{sec}$ after the final conditioning pulse. The data are plotted as the ratio of the size of the RRP when $3 \mu \mathrm{m}$ nicotine was included in the conditioning pulses to the size of the RRP when the conditioning pulses contained no nicotine. At 5 sec interpulse intervals, there was an initial increase and a subsequent decrease in the size of the RRP that decayed with a time constant of $9.0 \mathrm{sec}$. When $10 \mathrm{sec}$ intervals were used, the sucrose response increased as a function of the number of nicotine pulses; the data were fitted (smooth curve) using a single exponential with a maximal enhancement of $47.0 \%$ and a time constant of $33 \mathrm{sec}$. As indicated by the asterisks, the response recorded after 5 ( $p=0.011)$ and 10 ( $p=0.035)$ pulses were statistically significant. Data are the average of five experiments performed in quadruplicate. At 15 sec intervals, the size of the RRP increased monotonically, but the plateau value was lower than that observed with a $10 \mathrm{sec}$ interval. $B$, Recovery from nicotine-induced enhancement was determined by delivering five conditioning pulses as before and then delivering the hypertonic sucrose pulse at defined intervals after the final nicotine pulse (O). The recovery was rapid, with a time constant of $8.6 \mathrm{sec}$ based on an exponential decay to a plateau value of 1.014. The enhancement was statistically significant ( $p=0.004$ ) only for the shortest interpulse interval ( $1 \mathrm{sec}$ ). Data are plotted as averages of six experiments performed in quadruplicate.

$0.6 \mu \mathrm{M}$; in contrast, the potency of nicotine as a modulator of RRP size was approximately twofold less $\left(\mathrm{EC}_{50}\right.$ value of $\left.1.3 \mu \mathrm{M}\right)$. Although the observed difference in potency was not large, it was consistent with the lower potency of nicotine for $\alpha 7$ receptors. A decrease in efficacy of nicotine was consistently observed as concentrations were increased above $3 \mu \mathrm{M}$, such that little enhancement was observed at $100 \mu \mathrm{M}$. This observation is consistent with the presence of an acute desensitization of nAChRs at micromolar concentrations of nicotine that limit its apparent potency.

The kinetics of the nicotine response were examined by varying the number and frequency of the conditioning stimuli delivered before a sucrose test pulse (Fig. 5). A single $200 \mathrm{msec}$ pulse with $3 \mu \mathrm{M}$ nicotine slightly enhanced the sucrose response, an effect that was not statistically significant. If pulses were delivered at $5 \mathrm{sec}$ intervals, there was a similar small increase in the size of the RRP observed after the first pulse that subsequently decreased with additional stimuli, suggesting that $\mathrm{nAChR}$ desensitization predominates at this frequency. When stimulated at $10 \mathrm{sec}$ intervals, nicotine pulses enhanced the size of the RRP, an effect that increased to a plateau value of $47 \%$ within $2 \mathrm{~min}$. Delivering stimuli at $15 \mathrm{sec}$ intervals also increased the size of the RRP, but the plateau value of enhancement was only $24 \%$.

The duration of the enhancement was determined by delivering five $200 \mathrm{msec}$ nicotine pulses at $10 \mathrm{sec}$ intervals and then varying the interval between the fifth conditioning pulse and the sucrose test pulse. The decay of the enhancement could be ap- proximated by a first-order process with a time constant of 8.6 $\mathrm{sec}$, suggesting that this nicotine effect is short-lived.

\section{Choline selectively enhances the size of the RRP}

Choline, a precursor to and a hydrolytic product of acetylcholine, has been shown to be a selective (albeit low-potency) agonist of $\alpha 7$-containing nAChRs (Alkondon et al., 1997). The ability of $\alpha$ BgTx to block the nicotine-induced enhancement of the size of the RRP suggested that an $\alpha 7$-selective agonist such as choline should also produce enhancement. This possibility was tested using a two-pulse protocol consisting of a $7.5 \mathrm{sec}$ pulse with a stimulation buffer ( $3 \mu \mathrm{M}$ nicotine, $10 \mathrm{~mm}$ choline $\mathrm{Cl}$, or control saline), followed by a $9 \mathrm{sec}$ pulse with $0.5 \mathrm{M}$ sucrose saline to measure the RRP (Fig. 6). When the superfusate was switched from basal saline to a stimulus buffer that contained $2.5 \mathrm{mM} \mathrm{Ca}^{2+}$ (at $t=0)$, there was a transient increase in $\left[{ }^{3} \mathrm{H}\right]$ dopamine release even in the absence of a nicotinic agonist (Fig. $6 \mathrm{~A}$, inset). This transient release event has been observed previously (Bowyer and Weiner, 1990) and is probably dependent on $\mathrm{Ca}^{2+}$ entry via neuronal calcium channels via a poorly understood mechanism. When $3 \mu \mathrm{M}$ nicotine was included in the stimulus buffer, there was a modest increase in $\left[{ }^{3} \mathrm{H}\right]$ dopamine release that was insensitive to $100 \mathrm{~nm} \alpha \mathrm{BgTx}$, similar to the release event described in Figure 1. After exposing the synaptosomes to nicotine, the response to the second pulse containing hypertonic saline was significantly enhanced compared with saline control. This enhancement was essentially blocked by $100 \mathrm{nM} \alpha \mathrm{BgTx}$. In contrast, a 7.5 sec pulse with a stimulus buffer that contained $10 \mathrm{~mm}$ choline $\mathrm{Cl}$ had no measurable effect on the rate of $\left[{ }^{3} \mathrm{H}\right]$ dopamine release when compared with control. However, like nicotine, choline treatment produced a significant increase in the response to hypertonic saline that was eliminated by $100 \mathrm{~nm} \alpha \mathrm{BgTx}$. Thus, choline selectively enhanced the sucrose response without directly evoking $\left[{ }^{3} \mathrm{H}\right]$ dopamine release, consistent with a selective activation of $\alpha 7$-containing nAChRs and suggesting that $\alpha 7 \mathrm{nAChRs}$ are expressed at relatively low levels compared with non- $\alpha 7$ receptors.

To strengthen the pharmacological identity of the nAChR that mediated the actions of nicotine in this assay, the same experimental paradigm was repeated including dihydro- $\beta$ erythroidine (100 $\mathrm{nm}$ ), a selective inhibitor of $\beta 2$-containing nAChRs, or methyllycaconitine (30 nM), a high-potency inhibitor of $\alpha 7$ receptors, in the nicotine-containing stimulus buffer. Dihydro- $\beta$-erythroidine eliminated nicotine-evoked dopamine release without altering the enhancement of sucrose-evoked release; methyllycaconitine had the converse action (data not shown).

\section{Signaling pathways that couple nAChRs to changes in synaptic vesicle dynamics}

The observation that $\mathrm{Ca}^{2+}$ entry mediated by $\alpha 7$ nAChRs enhanced the response to hypertonic saline suggested that an intracellular calcium signaling pathways linked receptor activity with synaptic vesicle mobilization. An obvious candidate for an intracellular mediator of such a pathway was calmodulin, a prominent $\mathrm{Ca}^{2+}$ binding protein that is ubiquitous in the brain. A two-pulse protocol consisting of a sustained (7.5 sec duration) nicotine pulse followed immediately by a pulse with hypertonic saline (Fig. 7) was used to evaluate the role of calmodulin in the nicotine-induced enhancement. As described previously (Figs. 1, 6), sustained application of $3 \mu \mathrm{M}$ nicotine produced a biphasic increase in dopamine release rate. Subsequent application of hy- 

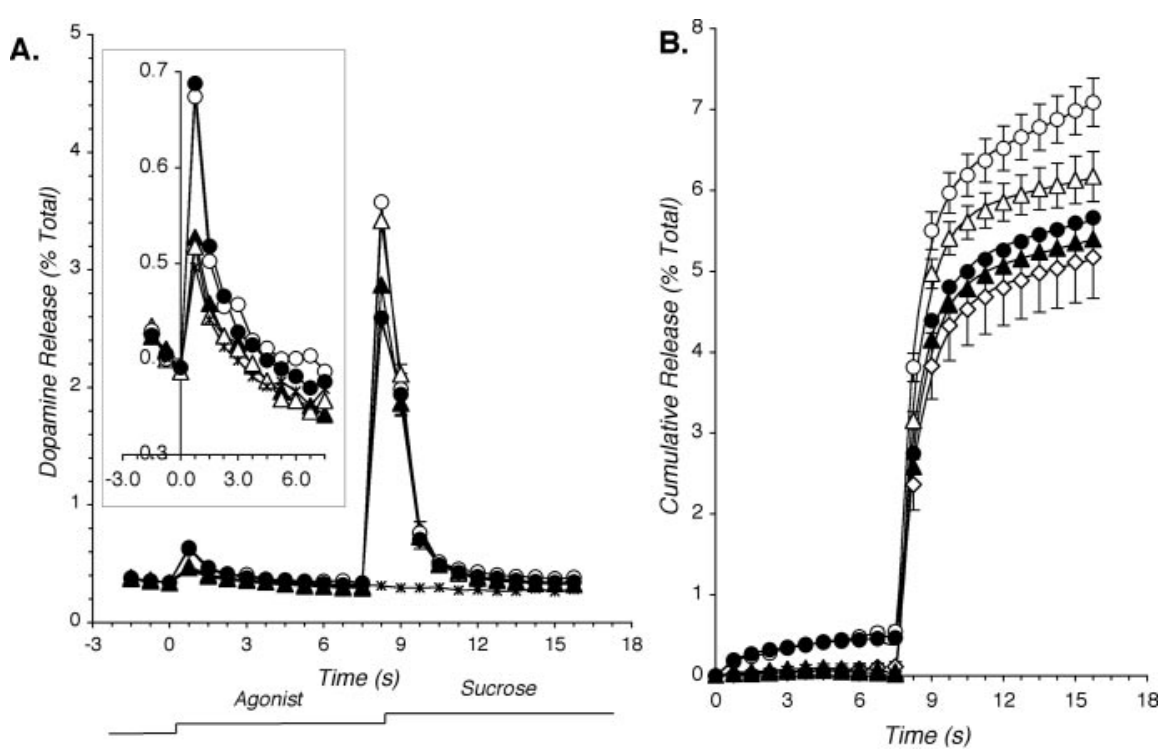

Figure 6. Choline selectively enhances the response to hypertonic sucrose. $A, A$ two-pulse protocol was used to test the ability of the $\alpha 7$-selective agonist choline to enhance the response to sucrose. A 7.5 sec conditioning pulse with saline ${ }^{*}$ ), $3 \mu \mathrm{m}$ nicotine $(\bigcirc), 3 \mu \mathrm{m}$ nicotine plus $100 \mathrm{~nm} \alpha \mathrm{BgTx}(\bullet), 10 \mathrm{~mm}$ choline $(\triangle)$, or $10 \mathrm{~mm}$ choline plus $\alpha \mathrm{Bg} \operatorname{Tx}(\boldsymbol{\Delta})$ was immediately followed by a 9.0 sec test pulse with saline containing $0.5 \mathrm{~m} \mathrm{sucrose}(\diamond)$ (or saline alone, ${ }^{*}$ ) to measure the size of the RRP. Nicotine and choline both enhanced the secretory response to hypetonic saline in an $\alpha \mathrm{Bg} \mathrm{Tx}$-sensitive manner. Inset, Expanded scale for the release rate during the conditioning pulse illustrates that nicotine-evoked dopamine release was insensitive to $100 \mathrm{~nm} \alpha \mathrm{Bg} T$; choline had no significant effect on dopamine release rates when compared with control (symbol use consistent with $A$ ). $B$, Net dopamine release measured in $A$ was integrated to reveal the cumulative action of nicotinic agonists on dopamine release. Symbols used are consistent with those in $A$. Smooth curves describe the nonlinear regression fit of each data set using the sum of a singleexponential component plus a linear component. Error bars represent the SEM for 19 measurements for each experimental condition performed on five separate synaptosome preparations. Cumulative release measured at the end of the test pulse after both the nicotine and the choline conditioning pulses was significantly different than for control ( $p<0.01$ and $p<0.03$, respectively; Student's two-tailed $t$ test). Error bars for nicotine plus $\alpha \mathrm{BgTx}$ and choline plus $\alpha \mathrm{Bg} \mathrm{Tx}$ were omitted for clarity.

pertonic saline revealed that, compared with control release rates, the nicotine pulse enhanced the amount of dopamine released.

When synaptosomes were pretreated with the calmodulin inhibitor calmidazolium, two prominent effects were observed (Fig. 7A). First, dopamine release evoked by the sustained application of nicotine was diminished, such that $300 \mathrm{~nm}$ calmidazolium selectively blocked the linear, $\mathrm{Na}^{+}$-independent component without altering the fast, exponential component. Second, the calmidazolium pretreatment blocked the nicotine enhancement of sucrose-evoked release. Control experiments (data not shown) demonstrated that $300 \mathrm{~nm}$ calmidazolium had no effect on sucrose-evoked release from synaptosomes that had not been stimulated with nicotine. It should be noted that these actions of calmidazolium were unlikely to be attributable to a direct blockade of the nAChRs because the synaptosomes were only pretreated with calmidazolium for 4 min immediately before the release experiment. This protocol allowed the drug to gain access to the cytoplasmic compartment, but external drug was removed by an extensive wash with basal saline that was administered immediately before the two-pulse protocol.

Because the selectivity of calmidazolium, even at submicromolar concentrations, is questionable, a second calmodulin inhibitor was tested. Trifluperazine also inhibits calmodulinmediated signaling, albeit at lower potency than calmidazolium. An examination of the effects of these two agents on nicotineinduced increases in the response to hypertonic sucrose indicated that calmidazolium was at least 10 -fold more potent than trifluperazine and that both agents were effective at submicromolar concentrations.
The selective calmodulin kinase inhibitor KN-62 (1-[NO-bis-1,5-isoquinolinesulfonyl]-N-methyl-L-tyrosyl-4-phenylpiperazine) was used to test the possible role of this kinase in mediating the actions of calmodulin (Fig. 7B). As with calmidazolium, KN-62 (1 $\mu \mathrm{M})$ blocked the linear component of nicotine-evoked dopamine release. However, at this concentration, KN-62 had no discernable effect on the sucroseevoked release. The results with calmidazolium and $\mathrm{KN}-62$, together with the inability of $\mathrm{Ba}^{2+}$ to substitute for $\mathrm{Ca}^{2+}$ in the nicotine enhancement of sucroseevoked release, indicate that $\mathrm{Ca}^{2+}$ entry through $\alpha 7 \mathrm{nAChRs}$ alters synaptic vesicle dynamics via a calmodulin-dependent signaling pathway that does not require calmodulin kinase activity. Furthermore, these results suggest that the linear phase of nicotine-evoked release may involve a calmodulin kinase-dependent mechanism, because both calmidazolium and KN-62 inhibited this process. Although the accuracy of conclusions made using a pharmacological approach is limited by the uncertain specificity of these drugs, it offers the ability to access intracellular signaling pathways that are otherwise difficult to study. More specific approaches will be required to help confirm the intracellular events that couple $\mathrm{Ca}^{2+}$ entry to synaptic vesicle mobilization in striatal dopamine terminals.

\section{Discussion}

Defining the role of nAChRs in the physiology and pharmacology of CNS neurons is an important task given the widespread distribution of cholinergic systems in the brain and the social significance of chronic tobacco use (McGehee and Role, 1995; Wonnacott, 1997). It is believed that cholinergic interneurons in the ventral striatum and cholinergic projection neurons that connect the pedunculopontine tegmental nucleus to the ventral tegmental area supply the acetylcholine that modulates presynaptic dopamine release and the firing of dopamine neurons, respectively. However, the physiological actions of nicotine appear to be quite complex and cannot be explained solely on the basis of acute activation of nAChRs. Chronic nicotine exposure, similar to what might be expected for smokers, primarily results in nAChR desensitization because the effect of nicotine is comparable with that of receptor antagonists, such as mecamylamine (Zhou et al., 2001). Furthermore, recent results obtained from striatal slices indicate that this desensitization may play an important role in the behavioral reinforcement observed for tobacco users. Rice and Cragg (2004) showed that nicotine suppressed striatal dopamine levels in response to low-frequency stimuli but augmented them at high $(25-100 \mathrm{~Hz})$ frequencies. Zhang and Sulzer (2004) demonstrated a similar frequency dependence of dopamine release and further showed that nicotine (or mecamylamine) could produce a short-term, $\mathrm{Ca}^{2+}$-dependent facilitation of electrically evoked dopamine release. The results reported here expand the complexities of presynaptic regulation of dopamine release prob- 
ability by invoking an $\alpha 7$-mediated regulated change in the size of the releasable pool of dopamine.

\section{Two distinct actions of nicotine found in striatal synaptosomes}

Biochemical measurement of radiolabeled dopamine release from mouse brain synaptosomes was used to examine the actions of nicotine on dopamine nerve terminals. These results confirm previous findings that nicotine stimulates dopamine release by activating presynaptic, non- $\alpha 7$ nAChRs. These receptors act to depolarize the terminals, triggering dopamine release by activating neuronal calcium channels. Those findings were extended by the demonstration that nicotine stimulated a $\mathrm{Ca}^{2+}$-dependent increase in synaptic vesicle availability, as assessed by the ability of hypetonic saline to evoke exocytosis of dopamine-containing vesicles. Unexpectedly, the ability of nicotine to modulate synaptic vesicle mobilization was blocked by $\alpha$ BgTx and methyllcaconitine but not by $5 \mu \mathrm{M}$ mecamylamine or dihydro- $\beta$-erythroidine, indicating that the ability of nicotine to enhance the response to hypertonic saline was mediated by an $\alpha 7$-containing nAChRs. This view was reinforced by the finding that choline, a selective $\alpha 7$ agonist, did not evoke $\left[{ }^{3} \mathrm{H}\right]$ dopamine release but selectively enhanced the response to sucrose in an $\alpha \mathrm{BgTx}$-sensitive manner.

Calmodulin (but not a CaM kinase) inhibitors selectively blocked the effects of nicotine on the response to hypertonic saline, suggesting a role for calmodulin in the signaling pathway. Both classes of inhibitor blocked the linear, or late, phase of nicotine-evoked release. Although this may represent a nonselective effect of these relatively hydrophobic agents, it is also consistent with a role for calmodulin-dependent kinase in nicotine-evoked release. Further discrimination between these possibilities will require additional work. Taken as a whole, the pharmacological results described in this study implicate $\mathrm{Ca}^{2+}$ entry mediated by presynaptic $\alpha 7$ $\mathrm{nAChRs}$ in the regulation of synaptic vesicle dynamics in dopamine terminals.

\section{Nicotine activates two distinct signaling pathways in striatal dopamine terminals}

The consensus view holds that presynaptic nAChRs regulating striatal dopamine release are non- $\alpha$, because nicotine-induced release is $\alpha \mathrm{BgTx}$ insensitive and $\alpha \mathrm{BgTx}$ binding site density is relatively low in this region. The evidence that $\alpha 7 \mathrm{nAChRs}$ on dopamine terminals can regulate synaptic vesicle dynamics adds to the complexity of presynaptic cholinergic mechanisms. Anatomical and pharmacological evidence in support of these findings has been reported recently. Reverse transcription-PCR amplification of RNA from individual cells in ventral midbrain that innervate the nucleus accumbens indicates that $\sim 40 \%$ of the tyrosine hydroxylase-positive dopamine cells express mRNA encoding $\alpha 7$ along with other more common nAChR subunits (Klink et al., 2001). Low levels of $\alpha 7$ nAChRs in the dopamine terminals suggested by cytological studies may indicate that the
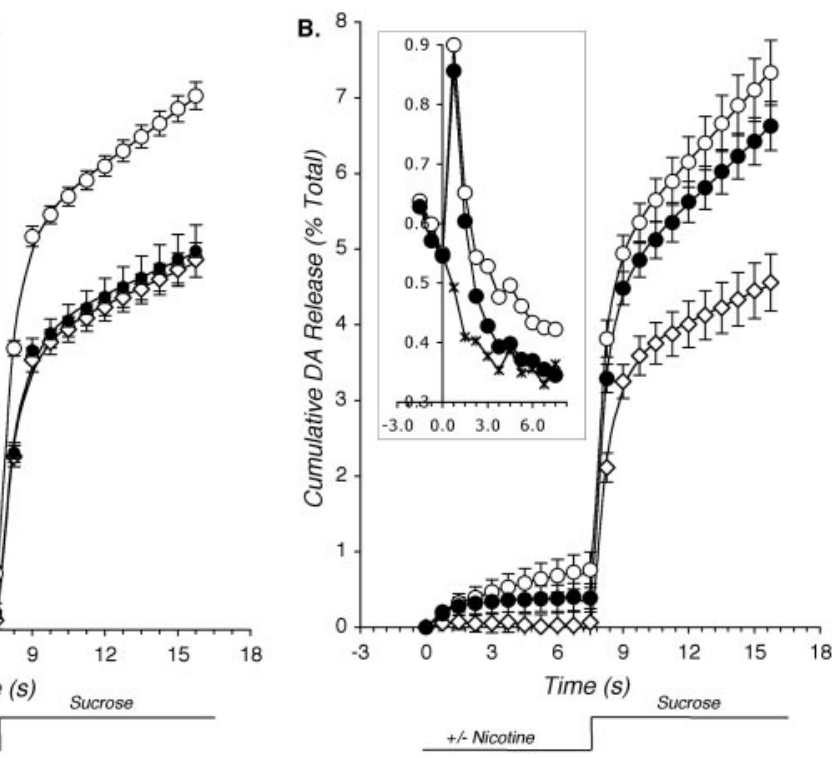

-pulse protocol described for Figure 6 was used to explore the signaling pathway that couples nAChRs to synaptic vesicle dynamics in dopamine terminals. $A$, Synaptosomes loaded with $\left.{ }^{3} \mathrm{H}\right]$ dopamine were given a conditioning pulse of basal saline $(\diamond)$, plus $3 \mu \mathrm{m}$ nicotine $(\bigcirc)$, or plus $3 \mu \mathrm{m}$ nicotine and $300 \mathrm{~nm}$ made using three different synaptosomal preparations. Cumulative dopamine release at the end of the experiment was significantly higher for the nicotine-only conditioning compared with the saline control ( $p<0.001$ and $p<0.03$, respectively, for $\mathrm{Ca}^{2+}$-dependent modulation of synaptic vesicle dynamics occurs via a tight "nanodomain" coupling of the $\alpha 7$ nAChRs into the signaling machinery at dopamine terminals and is consistent with the observation that the selective $\alpha 7 \mathrm{nAChR}$ agonist choline specifically enhanced synaptic vesicle availability without directly evoking exocytosis. Evidence that in situ application of $\alpha \mathrm{BgTx}$ (but not mecamylamine) into the nucleus accumbens suppressed nicotine-stimulated dopamine release (Fu et al., 2000) is consistent with this view.

Although it has been well documented that the $\alpha 7 \mathrm{nAChR}$ has a large $\mathrm{Ca}^{2+}$ permeability, there is surprisingly little evidence for a functional role for $\mathrm{Ca}^{2+}$ entry through the $\alpha 7 \mathrm{nAChRs}$ in neurons. $\alpha 7 \mathrm{nAChRs}$ have been shown to be necessary for reliable synaptic transmission during early development in the chick (Chang and Berg, 1999), and the same group has demonstrated $\alpha 7$-dependent calcium transients in dendritic spines (Shoop et al., 2001). The data presented here demonstrate that $\mathrm{Ca}^{2+}$ entry via $\alpha \mathrm{BgTx}$-sensitive nAChRs is selectively coupled to synaptic vesicle dynamics and that the signaling pathway that mediates this effect likely involves calmodulin. The ability of the $\alpha 7$ selective agonist choline to selectively enhance the sucroseevoked response without directly triggering dopamine release is consistent with the view that $\alpha 7 \mathrm{nAChRs}$ are relatively sparse in the dopamine terminals. Morphological evidence suggests that $\alpha 7 \mathrm{nAChR}$ targeting and localization is tightly specified by protein-protein interactions between the second intracellular loop of nAChRs and various cytoskeletal elements (Williams et al., 1998). Additional evidence supports the possibility that $\alpha 7$ $\mathrm{nAChRs}$ are localized in lipid raft signaling domains (Oshikawa et al., 2003; Roth and Berg, 2003). Together with the results in this 
report, it is reasonable to hypothesize that $\alpha 7 \mathrm{nAChRs}$ are specifically imbedded in a signaling complex responsible for maintaining synaptic vesicle availability by a calcium/calmodulindependent, positive feedback regulation of vesicle mobilization.

\section{Measuring the releasable vesicle pool with hypertonic saline}

The conclusions made regarding changes in the size of the RRP rely on the technique first described in the 1950s (Fatt and Katz, 1952; Furshpan, 1956) and more recently refined by Stevens and colleagues. The mechanisms by which hypertonic solutions promote vesicular release remain obscure but presumably involve mechanical forces imposed on the active zone by the gradient in osmotic pressure, perhaps by differential dehydration of the cytoplasmic space relative to the synaptic vesicle. With respect to this study, the relevant question is whether these observations of the action of hypertonic sucrose on synaptosomal $\left[{ }^{3} \mathrm{H}\right]$ dopamine release are a meaningful measure of the docked, fusioncompetent population of synaptic vesicles in dopamine terminals. Despite some obvious differences between the two systems, the similarities between these results and the sucrose-evoked glutamatergic currents in hippocampal autaptic neurons are striking. First, the time course of sucrose-evoked dopamine release from synaptosomes and the autaptic EPSP events in hippocampal neurons are nearly indistinguishable (Rosenmund and Stevens, 1996, compare their Figs. 1B, 3A). Second, phorbol esters promoted an increase in the size of the RRP and in the slope of the "linear" phase of release thought to reflect refilling rates in both cases. Phorbol treatment increased the size of the RRP by $57 \%$ in synaptosomes, comparable with the $65 \%$ increase reported previously (Stevens and Sullivan, 1998). Finally, reserpine, a selective inhibitor of the vesicular monoamine transporter, was used to demonstrate that osmotically evoked release of dopamine originated from a vesicular pool, because reserpine treatment reduced the size of the sucrose response by $71 \%$ but only diminished dopamine content by $36 \%$. The ability to make rapid measurements on transmitters such as dopamine that do not activate ionotropic receptors underscores the advantages of taking a biochemical approach to measuring radioisotope flux in striatal synaptosomes to study vesicle dynamics in monoaminergic terminals.

The regulation of synaptic vesicle dynamics by presynaptic signaling mechanisms has been studied extensively over the past decade. There is compelling evidence that the synaptic vesicle cycle is regulated at several steps and that multiple signaling pathways are involved. The size of the RRP of dopamine, and the rate of refilling of that pool, was increased by $\mathrm{PDBu}$ in a manner consistent with that described previously. Such results indicate that $\mathrm{Ca}^{2+}$ entry via the $\alpha 7 \mathrm{nAChR}$ regulates the RRP in a similar manner. The signaling pathways involved are yet to be delineated, but previous work on monoaminergic vesicles in chromaffin cells indicated that $\mathrm{Ca}^{2+}$ has multiple actions on vesicle dynamics in that system. Thus, there may be significant similarity between epinephrine-containing vesicles in the adrenal medulla and dopaminergic vesicles of nigral neurons.

\section{Physiological significance of transmitter regulation of synaptic vesicle dynamics}

These results support distinct roles for multiple subtypes of nAChRs in regulating striatal dopamine release. It is well established that non- $\alpha 7$ nAChRs trigger dopamine secretion by depolarization-dependent gating of exocytotic calcium channels. This report documents how presynaptic $\alpha 7 \mathrm{nAChRs}$ regulate the size of the RRP in a $\mathrm{Ca}^{2+}$-dependent manner. The observation that the $\alpha 7$-selective agonist choline actives this pathway suggests a novel mechanism for regulating the probability of dopamine release by increasing the supply of readily releasable vesicles. According to this view, acetylcholine, or its hydrolysis product choline, would activate presynaptic $\alpha 7$ receptors, leading to a calcium-dependent enhancement of the amount of dopamine available for release. This enhancement might be accomplished by increasing the rate of synaptic vesicle mobilization from a reserve pool, allowing them to dock and to be primed for release. Because of the relatively low potency of acetylcholine on $\alpha 7$ nAChRs, it is possible that the two activities described here act sequentially. At low levels of cholinergic stimulation, the non- $\alpha 7$ pathway predominates, promoting modest amounts of dopamine release attributable to increased calcium channel activity. At higher levels of acetylcholine release, $\alpha 7 \mathrm{nAChR}$-dependent increases in vesicle refilling could help replenish the RRP. Alternatively, extracellular choline produced by acetylcholinesterase could selectively activate the $\alpha 7$-dependent refilling pathway. Either of these scenarios could constitute a positive feedback loop opposing activity-dependent depletion, helping to maintain release probability under conditions of high exocytotic rates.

\section{References}

Alkondon M, Pereira EF, Cortes WS, Maelicke A, Albuquerque EX (1997) Choline is a selective agonist of alpha7 nicotinic acetylcholine receptors in the rat brain neurons. Eur J Neurosci 9:2734-2742.

Bowyer JF, Weiner N (1990) $\mathrm{Ca}^{2+}$-evoked $\left[{ }^{3} \mathrm{H}\right]$ dopamine release from synaptosomes is dependent on neuronal type $\mathrm{Ca}^{2+}$ channels and is not mediated by acetylcholine, glutamate or aspartate release. J Pharmacol Exp Ther 254:664-670.

Chang KT, Berg DK (1999) Nicotinic acetylcholine receptors containing $\alpha 7$ subunits are required for reliable synaptic transmission in situ. J Neurosci 19:3701-3710.

Clarke PBS, Reuben M (1996) Release of $\left[{ }^{3} \mathrm{H}\right]$ noradrenaline from rat hippocampal synaptosomes by nicotine: mediation by different nicotinic receptor subtypes from striatal $\left[{ }^{3} \mathrm{H}\right]$ dopamine release. Br J Pharmacol 117:595-606.

Dunkley PR, Jarvie PE, Heath JW, Kidd GJ, Rostas JAP (1986) A rapid method for isolation of synaptosomes on Percoll gradients. Brain Res 372:115-129.

Fatt P, Katz B (1952) Spontaneous subthreshold activity at motor nerve endings. J Physiol (Lond) 117:109-128.

Forbush B (1984) An apparatus for rapid kinetic analysis of isotope efflux from membrane vesicles and of ligand dissociation from membrane proteins. Anal Biochem 140:495-505.

Fu Y, Matta SG, Gao W, Sharp BM (2000) Local $\alpha$-bungarotoxin-sensitive nicotinic receptors in the nucleus accumbens modulate nicotinestimulated dopamine secretion in vivo. Neuroscience 101:369-375.

Furshpan EJ (1956) The effects of osmotic pressure changes on the spontaneous activity at motor nerve endings. J Physiol (Lond) 134:689-697.

Gillis KD, Mossner R, Neher E (1996) Protein kinase C enhances exocytosis from chromaffin cells by increasing the size of the readily releasable pool of secretory granules. Neuron 16:1209-1220.

Grady S, Marks MJ, Wonnacott S, Collins AC (1992) Characterization of nicotinic receptor-mediated $\left[{ }^{3} \mathrm{H}\right]$ dopamine release from synaptosomes prepared from mouse striatum. J Neurochem 59:848-856.

Gray R, Rajan AS, Radcliffe KA, Yakehiro M, Dani JA (1996) Hippocampal synaptic transmission enhanced by low concentrations of nicotine. Nature 383:713-716.

Klink R, de Kerchove d'Exaerde A, Zoli M, Changeux J-P (2001) Molecular and physiological diversity of nicotinic acetylcholine receptors in the midbrain dopaminergic nuclei. J Neurosci 21:1452-1463.

Koyama S, Matsumoto N, Kubo C, Akaike N (2000) Presynaptic 5-HT3 receptor-mediated modulation of synaptic GABA release in the mechanically dissociated rat amygdala neurons. J Physiol (Lond) 529:373-383.

Léna C, Changeux J-P (1997) Role of $\mathrm{Ca}^{2+}$ ions in nicotinic facilitation of GABA release in mouse thalamus. J Neurosci 17:576-585.

Lonart G, Südhof TC (2000) Assembly of SNARE core complexes occurs 
prior to neurotransmitter release to set the readily-releasable pool of synaptic vesicles. J Biol Chem 275:27703-27707.

MacDermott AB, Role LW, Siegelbaum SA (1999) Presynaptic ionotropic receptors and the control of transmitter release. Annu Rev Neurosci 22:443-485.

McGehee DS, Role LW (1995) Physiological diversity of nicotinic acetylcholine receptors expressed by vertebrate neurons. Annu Rev Physiol 57:521-546.

McQuiston AR, Madison DV (1999) Nicotinic receptor activation excites distinct subtypes of interneurons in the rat hippocampus. J Neurosci 19:2887-2896.

Miller RJ (1998) Presynaptic receptors. Annu Rev Pharmacol Toxicol 38:201-227.

Oshikawa J, Toya Y, Fujita T, Egawa M, Kawabe J, Umemura S, Ishikawa Y (2003) Nicotinic acetylcholine receptor alpha 7 regulates cAMP signal within lipid rafts. Am J Physiol Cell Physiol 285:C567-C574.

Prince RJ, Fernandes KG, Gregory JC, Martyn ID, Lippiello PM (1996) Modulation of nicotine-evoked $[3 \mathrm{H}]$ dopamine release from rat striatal synaptosomes by voltage-sensitive calcium channel ligands. Biochem Pharmacol 52:613-618.

Radcliffe KA, Dani JA (1998) Nicotinic stimulation produces multiple forms of increased glutamatergic synaptic transmission. J Neurosci 18:7075-7083.

Rapier C, Lunt GG, Wonnacott S (1988) Stereoselective nicotine-induced release of dopamine from striatal synaptosomes: concentration dependence and repetitive stimulation. J Neurochem 50:1123-1130.

Rice ME, Cragg SJ (2004) Nicotine amplifies reward-related dopamine signals in striatum. Nat Neurosci 7:583-584.

Rosenmund C, Stevens CF (1996) Definition of the readily releasable pool of vesicles at hippocampal synapses. Neuron 16:1197-1207.

Roth AL, Berg DK (2003) Large clusters of alpha7-containing nicotinic acetylcholine receptors on chick spinal cord neurons. J Comp Neurol 465:195-204.

Sands SB, Costa AC, Patrick JW (1993) Barium permeability of neuronal nicotinic receptor alpha 7 expressed in Xenopus oocytes. Biophys J 65:2614-2621.
Schuldiner S (1994) A molecular glimpse of vesicular monoamine transporters. J Neurochem 62:2067-2078.

Shoop RD, Chang KT, Ellisman MH, Berg DK (2001) Synaptically driven calcium transients via nicotinic receptors on somatic spines. J Neurosci 21:771-781.

Smith C, Moser T, Xu T, Neher E (1998) Cytosolic Ca ${ }^{2+}$ acts by two separate pathways to modulate the supply of release-competent vesicles in chromaffin cells. Neuron 20:1243-1253.

Stevens CF, Sullivan JM (1998) Regulation of the readily releasable vesicle pool by protein kinase C. Neuron 21:885-893.

Stevens CF, Tsujimoto T (1995) Estimates for the pool size of releasable quanta at a single central synapse and for the time required to refill the pool. Proc Natl Acad Sci USA 92:846-849.

Stevens CF, Wesseling JF (1998) Activity-dependent modulation of the rate at which synaptic vesicles become available to undergo exocytosis. Neuron 21:415-424.

Turner TJ, Goldin SM (1989) Multiple components of synaptosomal $\left[{ }^{3} \mathrm{H}\right]$ $\gamma$-aminobutyric acid release resolved by a rapid superfusion system. Biochemistry 28:586-593.

Turner TJ, Pearce LB, Goldin SM (1989) A superfusion system designed to measure release of radiolabeled neurotransmitters on a subsecond time scale. Anal Biochem 178:8-16.

Turner TJ, Adams ME, Dunlap K (1993) Multiple $\mathrm{Ca}^{2+}$ channel types coexist to regulate synaptosomal neurotransmitter release. Proc Natl Acad Sci USA 90:9518-9522.

Williams BM, Temburni MK, Levey MS, Bertrand S, Bertrand D, Jacob MH (1998) The long internal loop of the alpha 3 subunit targets nAChRs to subdomains within individual synapses on neurons in vivo. Nat Neurosci 1:557-562.

Wise RA, Rompre P-P (1989) Brain dopamine and reward. Annu Rev Psychol 40:191-225.

Wonnacott S (1997) Presynaptic nicotinic ACh receptors. Trends Neurosci 20:92-98.

Zhang H, Sulzer D (2004) Frequency-dependent modulation of dopamine release by nicotine. Nat Neurosci 7:581-582.

Zhou FM, Liang Y, Dani JA (2001) Endogenous nicotinic cholinergic activity regulates dopamine release in the striatum. Nat Neurosci 4:1224-1229. 